\title{
Catalogue of Balanced Repeated Measurement Designs in Linear Periods of Two Unequal Sizes
}

\author{
Muhammad Amin Khalid, Muhammad Zahid Bashir and Rashid Ahmed* \\ Department of Statistics, Allama Iqbal Open University Islamabad, Pakistan
}

Submission: March 19, 2018; Published: July 09, 2018

*Corresponding author: Rashid Ahmed, Department of Statistics, TheIslamia University of Bahawalpur, Pakistan, Email: rashid701@hotmail.com

Abstract

Balanced repeated measurement designs have their own importance to control the carry over or residual effects. There are many situations when experiments become infeasible to perform in periods of equal sizes. Then these designs should be used in periods of unequal sizes. In this study, some useful balanced and strongly balanced repeated measurement designs are presented in periods of two unequal sizes.

Keywords: Repeated measurement designs; Circular balanced repeated measurement designs; Circular strongly balanced repeated measurement designs; Method of cyclic shifts; Repeated measurement; Residual effect; Subclass of designs; Hamiltonian decomposition; Lexicographic; Two graphs; Design and analysis; Cross-over trials; Balanced RMDs; Linear periods

Abbreviations: RMD: Repeated Measurement Design; CSBRMD: Circular Strongly Balanced Repeated Measurements Designs; CBRMD: Circular Balanced Repeated Measurements Designs

\section{Introduction}

A repeated measurement design (RMD) is one in which multiple, or repeated measurements are made on each experimental unit. The experimental unit could be a person or an animal. In repeated measurement design each subject is measured before and one or several times after an intervention. Areas where RMDs are widely used include medicine, pharmacology, animal sciences and psychology. A characteristic feature of RMD is that the effect which a treatment has during its period of application (its direct effect) may persist into the following period(s). If the effect persists only into the immediately following period, the effect is called the first-order residual effect or residual effect. The choice of RMD must be made in a way that the treatments can be efficiently compared after allowing for the residual effects. In this paper circular balanced and strongly balanced repeated measurement designs are constructed to extend the work of Iqbal and Tahir [1] for $\mathrm{v} \leq 100$ in 3 periods.

Williams [2,3] first initiated repeated measurements designs. Magda [4] introduced the idea of a circular balance repeated measurement design when proper balance for different effects is considered, also proved its universal optimality over the subclass of designs with the same set of parameters. Roy [5] constructed strongly balanced uniform repeated measurements designs for $\mathrm{v}=0,1$ or 3 modulo 4 , by using the methods of differences and Hamiltonian decomposition of the lexicographic product of two graphs. Jones \& Kenward [6] gave a thorough review of the design and analysis of cross-over trials. They also discussed its importance. Afsarinejed [7] presented some construction methods for repeated measurement design. Cheng \& Wu [8] explained two different types of repeated measurements designs (RMD), the balanced uniform RMD and the strongly balanced uniform RMD. Iqbal \& Jones [9] constructed

i. Efficient RMDs with equal and unequal period sizes using method of cyclic shifts for $3 \leq v \leq 10,3 \leq p \leq 10$,

ii. Strongly balanced RMDs for $3 \leq v \leq 10,3 \leq p \leq 10$,

iii. RMDs that are balanced for first and second order residual effect for $6 \leq v \leq 9,4 \leq p \leq 6$ and

iv. combinatorial balanced designs for two un equal number of periods for $5 \leq v \leq 10,3 \leq p_{1} \leq 6$ and $3 \leq p_{2} \leq 10$.

Sharma et al. [10] introduced a general strategy of construction of balanced RMDs for odd number of treatments and their analysis. Iqbal\& Tahir [1] constructed CSBRMD (circular strongly balanced repeated measurements designs) for some classes. Iqbal et al. [11] constructed some first- and second-order CBRMD (circular balanced repeated measurements designs). They also constructed some CSBRMDs. In this article, BRMDs and SBRMDs are constructed for linear periods in two different period sizes.

Definition 1.1: The set of all RMD with $p$ periods, $n$ experimental units and $v$ treatments is denoted by RM $(v, n, p)$. A repeated measurements design is balanced with respect to the first-order residual effects if each treatment is immediately preceded $\lambda^{\prime}$ times by each other treatment (excluding itself). 
Definition 1.2: A repeated measurements design is strongly balanced with respect to the first-order residual effects if each treatment is immediately preceded $\lambda^{\prime}$ times by each other treatment (including itself).

Definition 1.3: For given $v$ and $p$, a balanced or strongly balanced repeated measurements design is minimal if $\lambda^{\prime}=1$.

In this paper, method of cyclic shifts is explained briefly in Section 2. BRMDs and SBRMDs are constructed in Section 3 and 4 respectively.

\section{Method of Cyclic Shifts}

Method of cyclic shifts is explained here briefly. For detail, see Iqbal and Tahir [1] and Iqbal et al. [11].

\section{BRM Designs in Linear Blocks of two unequal Sizes}

Rule I: Let $S=\left[q_{1}, q_{2}, \ldots, q_{p-1}\right]$ be a set of shifts, where $1 \leq q_{i} \leq v-1$. If each element $1,2, \ldots, v-1$ appears an equal number of times, say $\lambda^{\prime}$ in a new set of shifts $S^{*}=\left[q_{1}, q_{2}, \ldots, q_{p-1}\right]$ then it will be BRMD. If $0 \leq q_{i} \leq v-1$ and each element $0,1, \ldots, v-1$ appears an equal number of times, say $\lambda^{\prime}$ in a new set of shifts $S *$ then it will be SBRMD [12].

Rule II: Let $S=\left[q_{1}, q_{2}, \ldots, q_{(p-2)}\right] t$ be a set of shifts, where $1 \leq q_{i} \leq v-2$. If each element $1,2, \ldots, v-2$ appears an equal number of times, say $\lambda^{\prime}$ in a new set of shifts $S^{*}=\left[q_{1}, q_{2}, \ldots, q_{(p-2)}\right]$ then it will be BRMD. If $0 \leq q_{i} \leq v-2$ and each element $0,1, \ldots, v-2$ appears an equal number of times, say $\lambda^{\prime}$ in a new set of shifts $S *$ then it will be SBRMD.

In this Section BRMDs are constructed for linear periods two different sizes (Table 1,2).

Table 1: BRMDs for $p_{1}=5$ and $p_{2}=3$ (Linear Periods).

\begin{tabular}{|c|c|c|c|}
\hline$v$ & $p_{1}$ & $p_{2}$ & Sets of Shifts \\
\hline 7 & 5 & 3 & {$[1,3,2,4]+[5,6]$} \\
\hline 9 & 5 & 3 & {$[1,2,3,5]+[4,6]+[7,8]$} \\
\hline 11 & 5 & 3 & {$[1,2,3,4]+[5,7,6,8]+[9,10]$} \\
\hline 13 & 5 & 3 & {$[1,2,3,4]+[5,6,8,9]+[7,10]+[11,12]$} \\
\hline 15 & 5 & 3 & {$[1,2,3,4]+[5,6,7,9]+[8,10,11,12]+[13,14]$} \\
\hline 17 & 5 & 3 & {$[1,2,3,4]+[5,6,7,8]+[9,10,11,12]+[13,14]+[15,16]$} \\
\hline 19 & 5 & 3 & {$[1,2,3,4]+[5,6,7,8]+[9,11,10,12]+[13,14,15,16]+[17,18]$} \\
\hline 21 & 5 & 3 & {$[1,2,3,4]+[6,5,7,8]+[9,10,13,11]+[12,14,15,16]+[17,18]+[19,20]$} \\
\hline 23 & 5 & 3 & {$[1,2,3,4]+[6,5,7,8]+[9,10,11,13]+[12,14,15,16]+[17,18,19,20]+[21]+[22]$} \\
\hline 25 & 5 & 3 & {$[1,2,3,4]+[6,5,7,8]+[9,10,11,12]+[13,14,15,16]+[17,18,19,20]+[21,22]+[23,24]$} \\
\hline 27 & 5 & 3 & {$[1,2,3,4]+[6,5,7,8]+[9,10,11,12]+[13,15,14,16]+[17,18,20,19]+[21,22,23,24]+[25,26]$} \\
\hline 29 & 5 & 3 & {$[1,2,3,4]+[6,5,7,8]+[9,10,11,12]+[13,14,17,15]+[16,18,20,19]+[21,22,23,24]+[25,26]+[27,28]$} \\
\hline 31 & 5 & 3 & {$[1,2,3,4]+[6,5,7,8]+[9,10,11,12]+[14,13,17,15]+[16,18,20,19]+[21,22,23,24]+[25,26,27,28]+[29,30]$} \\
\hline 33 & 5 & 3 & {$[1,2,3,4]+[6,5,7,8]+[11,9,10,12]+[13,14,17,15]+[16,18,20,19]+[21,22,24,23]+[25,26,27,28]+[29,30]+[31,32]$} \\
\hline 35 & 5 & 3 & {$[1,2,3,4]+[6,5,7,8]+[9,11,10,12]+[13,14,17,15]+[16,18,20,19]+[21,22,23,24]+[25,26,27,28]+[29,30,31,32]+[33,34]$} \\
\hline 37 & 5 & 3 & {$[1,2,3,4]+[6,5,7,8]+[9,11,10,12]+[13,14,17,15]+[16,18,20,19]+[21,22,23,24]+[25,26,27,28]+[29,30,31,32]+[33,34]+[35,36]$} \\
\hline 39 & 5 & 3 & {$[1,2,3,4]+[6,5,7,8]+[9,11,10,12]+[13,14,17,15]+[16,19,18,20]+[21,22,23,24]+[25,26,28,27]+[29,30,31,32]+[33,34,35,36]+[37]+[38]$} \\
\hline 41 & 5 & 3 & $\begin{array}{l}{[1,2,3,4]+[6,5,7,8]+[9,11,10,12]+[13,14,17,15]+[16,19,18,20]+[21,22,23,24]+[25,26,27,28]+[29,30,31,32]+[33,34,35,36]+[37,38]+[} \\
39,40]\end{array}$ \\
\hline 43 & 5 & 3 & $\begin{array}{l}{[1,2,3,4]+[6,5,7,8]+[9,11,10,12]+[13,14,17,15]+[16,19,18,20]+[21,22,23,24]+[25,26,27,28]+[29,30,31,32]+[33,34,35,36]+[37,38,39,} \\
40]+[41,42]\end{array}$ \\
\hline 45 & 5 & 3 & $\begin{array}{l}{[1,2,3,4]+[6,5,7,8]+[9,11,10,12]+[13,14,15,17]+[16,19,18,20]+[21,22,23,25]+[24,26,27,28]+[29,30,32,31]+[33,34,35,36]+[37,38,39,} \\
40]+[41,42]+[43,44]\end{array}$ \\
\hline 47 & 5 & 3 & $\begin{array}{l}{[1,2,3,4]+[6,5,7,8]+[9,11,10,12]+[13,14,15,16]+[17,19,18,20]+[21,22,23,25]+[24,26,27,28]+[29,30,32,31]+[33,34,35,36]+[37,38,39,} \\
40]+[41,42,43,44]+[45,46]\end{array}$ \\
\hline 49 & 5 & 3 & $\begin{array}{l}{[1,2,3,4]+[6,5,7,8]+[9,11,10,12]+[13,14,15,16]+[17,19,18,20]+[21,22,23,25]+[24,26,27,28]+[29,30,32,31]+[33,34,35,36]+[37,38,39,} \\
40]+[41,42,43,44]+[45,46]+[47,48]\end{array}$ \\
\hline
\end{tabular}

Table 2: BRMDs for $p_{1}=5$ and $p_{2}=4$ (Linear Periods).

\begin{tabular}{|c|c|c|l|}
\hline$v$ & $p_{1}$ & $p_{2}$ & Sets of Shifts \\
\hline 8 & 5 & 4 & {$[1,2,3,4]+[5,6,7]$} \\
\hline 11 & 5 & 4 & {$[1,2,3,4]+[5,7,6]+[8,9,10]$} \\
\hline
\end{tabular}




\section{Biostatistics and Biometrics Open Access Journal}

\begin{tabular}{|c|c|c|c|}
\hline 12 & 5 & 4 & {$[1,2,3,4]+[5,6,7,8]+[9,10,11]$} \\
\hline$v$ & $p_{1}$ & $p_{2}$ & Sets of Shifts \\
\hline 14 & 5 & 4 & {$[1,2,3,4]+[5,6,7]+[8,9,10]+[11,13,12]$} \\
\hline 15 & 5 & 4 & {$[1,2,3,4]+[5,6,7,9]+[8,10,11]+[12,13,14]$} \\
\hline 16 & 5 & 4 & {$[1,2,3,4]+[5,6,7,8]+[9,10,11,12]+[13,14,15]$} \\
\hline 17 & 5 & 4 & {$[1,2,3,4]+[5,6,7]+[8,9,10]+[11,12,13]+[14,15,16]$} \\
\hline 18 & 5 & 4 & {$[1,2,3,4]+[5,6,8,7]+[9,10,11]+[12,13,14]+[15,16,17]$} \\
\hline 19 & 5 & 4 & {$[1,2,3,4]+[5,6,7,8]+[9,11,10,12]+[13,14,15]+[16,17,18]$} \\
\hline 20 & 5 & 4 & {$[1,2,3,4]+[5,6,7,8]+[9,10,11,12]+[13,14,15,16]+[17,18,19]$} \\
\hline 21 & 5 & 4 & {$[1,2,3,4]+[6,5,7,8]+[9,10,13]+[11,12,14]+[15,16,17]+[18,19,20]$} \\
\hline 22 & 5 & 4 & {$[1,2,3,4]+[6,5,7,8]+[9,10,11,13]+[12,14,15]+[16,17,18]+[19,20,21]$} \\
\hline 23 & 5 & 4 & {$[1,2,3,4]+[6,5,7,8]+[9,10,11,13]+[12,14,15,16]+[17,18,19]+[20,21,22]$} \\
\hline 24 & 5 & 4 & {$[1,2,3,4]+[6,5,7,8]+[9,10,11,12]+[13,14,15,16]+[17,18,19,20]+[21,22,23]$} \\
\hline 25 & 5 & 4 & {$[1,2,3,4]+[6,5,7,8]+[9,10,11,12]+[13,14,15]+[16,17,18]+[19,20,21]+[22,23,24]$} \\
\hline 26 & 5 & 4 & {$[1,2,3,4]+[6,5,7,9]+[8,10,11,12]+[13,14,15,16]+[17,18,19]+[20,21,22]+[23,24,25]$} \\
\hline 27 & 5 & 4 & {$[1,2,3,4]+[6,5,7,8]+[9,10,11,12]+[13,15,14,16]+[17,18,20,19]+[21,22,23]+[24,25,26]$} \\
\hline 28 & 5 & 4 & {$[1,2,3,4]+[6,5,7,8]+[9,10,11,12]+[13,14,15,16]+[17,18,20,19]+[21,22,23,24]+[25,26,27]$} \\
\hline 29 & 5 & 4 & {$[1,2,3,4]+[6,5,7,8]+[9,10,11,12]+[13,14,17,15]+[16,18,20]+[19,21,22]+[23,24,25]+[26,27,28]$} \\
\hline 30 & 5 & 4 & {$[1,2,3,4]+[6,5,7,8]+[9,10,12,11]+[13,14,17,15]+[16,18,20,19]+[21,22,23]+[25,24,26]+[27,28,29]$} \\
\hline 31 & 5 & 4 & {$[1,2,3,4]+[6,5,7,8]+[9,10,11,12]+[14,13,17,15]+[16,18,20,19]+[21,22,23,24]+[25,26,27]+[28,29,30]$} \\
\hline 32 & 5 & 4 & {$[1,2,3,4]+[6,5,7,8]+[9,10,11,12]+[15,14,17,13]+[16,18,20,19]+[21,22,23,24]+[25,26,27,28]+[29,30,31]$} \\
\hline 33 & 5 & 4 & {$[1,2,3,4]+[6,5,7,8]+[11,9,10,12]+[13,14,17,15]+[16,18,20,19]+[21,22,24]+[23,25,26]+[27,28,29]+[30,31,32]$} \\
\hline 34 & 5 & 4 & {$[1,2,3,4]+[6,5,7,8]+[9,11,10,12]+[13,14,17,15]+[16,20,18,19]+[21,22,23,24]+[25,26,27]+[28,29,30]+[31,32,33]$} \\
\hline 35 & 5 & 4 & {$[1,2,3,4]+[6,5,7,8]+[9,11,10,12]+[13,14,17,15]+[16,18,20,19]+[21,22,23,24]+[25,26,27,28]+[29,30,31,32]+[33]+[34]$} \\
\hline 36 & 5 & 4 & {$[1,2,3,4]+[6,5,7,8]+[9,11,10,12]+[13,14,17,15]+[16,18,20,19]+[21,22,23,24]+[25,26,27,28]+[29,30,31,32]+[33,34,35]$} \\
\hline 37 & 5 & 4 & {$[1,2,3,4]+[6,5,7,8]+[9,11,10,12]+[13,14,17,15]+[16,18,20,19]+[21,22,23,24]+[25,26,27]+[28,29,30]+[31,32,33]+[34,35,36]$} \\
\hline 38 & 5 & 4 & {$[1,2,3,4]+[6,5,7,8]+[9,11,10,12]+[13,14,17,15]+[16,18,19,20]+[21,22,23,24]+[25,26,27,28]+[29,30,31]+[32,33,34]+[35,36,37]$} \\
\hline 39 & 5 & 4 & {$[1,2,3,4]+[6,5,7,8]+[9,11,10,12]+[13,14,17,15]+[16,19,18,20]+[21,22,23,24]+[25,26,28,27]+[29,30,31,32]+[33,34,35]+[36,37,38]$} \\
\hline 40 & 5 & 4 & {$[1,2,3,4]+[6,5,7,8]+[9,11,10,12]+[13,14,17,15]+[16,19,18,20]+[21,22,23,24]+[25,26,27,28]+[29,30,31,32]+[33,34,35,36]+[37,38,39]$} \\
\hline 41 & 5 & 4 & $\begin{array}{l}{[1,2,3,4]+[6,5,7,8]+[9,11,10,12]+[13,14,17,15]+[16,19,18,20]+[21,22,23,24]+[25,26,27,28]+[29,30,31]+[32,33,34]+[35,36,37]+[38,3} \\
9,40]\end{array}$ \\
\hline$v$ & $p_{1}$ & $p_{2}$ & Sets of Shifts \\
\hline 42 & 5 & 4 & $\begin{array}{l}{[1,2,3,4]+[6,5,7,8]+[9,11,10,13]+[12,14,17,15]+[16,19,18,20]+[21,22,23,24]+[25,26,27,28]+[29,30,31,32]+[33,34,35]+[36,37,38]+[3} \\
9,40,41]\end{array}$ \\
\hline 43 & 5 & 4 & $\begin{array}{l}{[1,2,3,4]+[6,5,7,8]+[9,11,10,12]+[13,14,17,15]+[16,19,18,20]+[21,22,23,24]+[25,26,27,28]+[29,30,31,32]+[33,34,35,36]+[37,38,39]} \\
+[40,41,42]\end{array}$ \\
\hline 44 & 5 & 4 & $\begin{array}{l}{[1,2,3,4]+[6,5,7,8]+[9,11,10,12]+[13,14,15,16]+[17,19,18,20]+[21,22,23,24]+[25,26,27,28]+[29,30,31,32]+[33,34,35,36]+[37,38,39,} \\
40]+[41,42,43]\end{array}$ \\
\hline 45 & 5 & 4 & $\begin{array}{l}{[1,2,3,4]+[6,5,7,8]+[9,11,10,12]+[13,14,15,17]+[16,19,18,20]+[21,22,23,25]+[24,26,27,28]+[29,30,32,31]+[33,34,35]+[36,37,38]+[3} \\
9,40,41]+[42,43,44]\end{array}$ \\
\hline 46 & 5 & 4 & $\begin{array}{l}{[1,2,3,4]+[6,5,7,8]+[9,11,10,12]+[13,14,15,16]+[17,19,18,20]+[21,22,23,25]+[24,26,27,28]+[29,30,32,31]+[33,34,35,37]+[36,38,39]} \\
+[40,41,42]+[43,44,45]\end{array}$ \\
\hline 47 & 5 & 4 & $\begin{array}{l}{[1,2,3,4]+[6,5,7,8]+[9,11,10,12]+[13,14,15,16]+[17,19,18,20]+[21,22,23,25]+[24,26,27,28]+[29,30,32,31]+[33,34,35,36]+[37,38,39,} \\
40]+[41,42,43]+[44,45,46]\end{array}$ \\
\hline 48 & 5 & 4 & $\begin{array}{l}{[1,2,3,4]+[6,5,7,8]+[9,11,10,12]+[13,14,15,16]+[17,19,18,20]+[21,22,23,25]+[24,26,27,28]+[29,30,32,31]+[33,34,35,36]+[37,38,39,} \\
40]+[41,42,43,44]+[45,46,47]\end{array}$ \\
\hline 49 & 5 & 4 & $\begin{array}{l}{[1,2,3,4]+[6,5,7,8]+[9,11,10,12]+[13,14,15,16]+[17,19,18,20]+[21,22,23,25]+[24,26,27,28]+[29,30,32,31]+[33,34,35,36]+[37,38,39]} \\
+[40,41,42]+[43,44,45]+[46,47,48]\end{array}$ \\
\hline 50 & 5 & 4 & $\begin{array}{l}{[1,2,3,4]+[6,5,7,8]+[9,11,10,12]+[13,14,15,16]+[17,19,18,20]+[21,22,23,25]+[24,26,27,28]+[29,30,32,31]+[33,34,35,36]+[37,38,39,} \\
40]+[41,42,43]+[44,45,46]+[47,48,49]\end{array}$ \\
\hline
\end{tabular}




\section{Biostatistics and Biometrics Open Access Journal}

\section{SBRM Designs in Linear Blocks of two unequal Sizes}

In this Section SBRMDs are constructed for linear periods two different sizes (Table 3,4).

Table 3: BRMDs for $p_{1}=5$ and $p_{2}=3$ (Linear Periods).

\begin{tabular}{|c|c|c|c|}
\hline$v$ & $p_{1}$ & $p_{2}$ & Sets of Shifts \\
\hline 6 & 5 & 3 & {$[0,1,3,2]+[4,5]$} \\
\hline 8 & 5 & 3 & {$[0,1,2,3]+[4,5]+[6,7]$} \\
\hline 10 & 5 & 3 & {$[0,1,2,3]+[4,5,6,7]+[8,9]$} \\
\hline 12 & 5 & 3 & {$[0,1,2,3]+[4,5,6,7]+[8,9]+[10,11]$} \\
\hline 14 & 5 & 3 & {$[0,1,2,3]+[4,5,6,7]+[8,9,10,11]+[12,13]$} \\
\hline 16 & 5 & 3 & {$[0,1,2,3]+[4,5,6,7]+[8,9,10,11]+[12,13]+[14,15]$} \\
\hline 18 & 5 & 3 & {$[0,1,2,3]+[5,4,6,7]+[8,9,10,11]+[12,13,14,15]+[16,17]$} \\
\hline 20 & 5 & 3 & {$[0,1,2,3]+[5,4,6,7]+[8,9,10,11]+[12,13,14,15]+[16,17]+[18,19]$} \\
\hline 22 & 5 & 3 & {$[0,1,2,3]+[5,4,6,8]+[7,9,10,11]+[12,13,14,15]+[16,17,18,19]+[20,21]$} \\
\hline 24 & 5 & 3 & {$[0,1,2,3]+[5,4,6,7]+[8,9,10,11]+[12,13,14,15]+[16,17,18,19]+[20,21]+[22,23]$} \\
\hline 26 & 5 & 3 & {$[0,1,2,3]+[5,4,6,7]+[8,9,10,11]+[12,13,14,15]+[16,17,18,19]+[20,21,22,23]+[24,25]$} \\
\hline 28 & 5 & 3 & {$[0,1,2,3]+[5,4,6,7]+[8,9,10,11]+[12,13,14,15]+[16,17,18,19]+[20,21,22,23]+[24,25]+[26,27]$} \\
\hline$v$ & $p_{1}$ & $p_{2}$ & Sets of Shifts \\
\hline 30 & 5 & 3 & {$[0,1,2,3]+[5,4,6,7]+[9,8,10,11]+[12,13,14,15]+[16,17,18,19]+[20,21,22,23]+[24,25,26,27]+[28,29]$} \\
\hline 32 & 5 & 3 & {$[0,1,2,3]+[5,4,6,7]+[8,9,10,11]+[12,13,14,15]+[16,17,18,19]+[20,21,22,23]+[24,25,26,27]+[28,29]+[30,31]$} \\
\hline 34 & 5 & 3 & {$[0,1,2,3]+[5,4,6,7]+[8,9,10,11]+[12,13,14,15]+[16,17,18,19]+[20,21,22,23]+[24,25,26,28]+[27,29,30,31]+[32,33]$} \\
\hline 36 & 5 & 3 & {$[0,1,2,3]+[5,4,6,7]+[8,9,10,11]+[12,13,14,15]+[16,17,18,19]+[20,21,22,23]+[24,25,26,27]+[28,29,30,31]+[32,33]+[34,35]$} \\
\hline 38 & 5 & 3 & {$[0,1,2,3]+[5,4,6,7]+[8,9,10,12]+[11,13,14,15]+[16,17,18,19]+[20,21,22,23]+[24,25,26,27]+[28,29,30,31]+[32,33,34,35]+[36,37]$} \\
\hline 40 & 5 & 3 & $\begin{array}{l}{[0,1,2,3]+[5,4,6,7]+[8,9,10,11]+[12,13,14,15]+[16,17,18,19]+[20,21,22,23]+[24,25,26,27]+[28,29,30,31]+[32,33,34,35]+[36,37} \\
]+[38,39]\end{array}$ \\
\hline 42 & 5 & 3 & $\begin{array}{l}{[0,1,2,3]+[5,4,6,7]+[8,9,10,11]+[13,12,14,15]+[16,17,18,19]+[20,21,22,23]+[24,25,26,27]+[28,29,30,31]+[32,33,34,35]+[36,37} \\
, 38,39]+[40,41]\end{array}$ \\
\hline 44 & 5 & 3 & $\begin{array}{l}{[0,1,2,3]+[5,4,6,7]+[8,9,10,11]+[12,13,14,15]+[16,17,18,19]+[20,21,22,23]+[24,25,26,27]+[28,29,30,31]+[32,33,34,35]+[36,37,} \\
38,39]+[40,41]+[42,43]\end{array}$ \\
\hline 46 & 5 & 3 & $\begin{array}{l}{[0,1,2,3]+[5,4,6,7]+[8,9,10,11]+[12,13,14,15]+[16,17,18,19]+[20,21,22,23]+[24,25,26,27]+[28,29,30,31]+[32,33,34,35]+[36,37} \\
38,39]+[40,41,42,43]+[44,45]\end{array}$ \\
\hline 48 & 5 & 3 & $\begin{array}{l}{[0,1,2,3]+[5,4,6,7]+[8,9,10,11]+[12,13,14,15]+[16,17,18,19]+[20,21,22,23]+[24,25,26,27]+[28,29,30,31]+[32,33,34,35]+[36,37,} \\
38,39]+[40,41,42,43]+[44,45]+[46,47]\end{array}$ \\
\hline 50 & 5 & 3 & $\begin{array}{l}{[0,1,2,3]+[5,4,6,7]+[8,9,10,11]+[12,13,14,15]+[16,17,18,19]+[20,21,22,23]+[24,25,26,27]+[28,29,30,31]+[32,33,34,35]+[36,37,} \\
38,40]+[39,41,42,43]+[44,45,46,47]+[48,49]\end{array}$ \\
\hline
\end{tabular}

Table 4: BRMDs for $p_{1}=5$ and $p_{2}=4$ (Linear Periods).

\begin{tabular}{|c|c|c|l|}
\hline$v$ & $p_{1}$ & $p_{2}$ & Sets of Shifts \\
\hline 7 & 5 & 4 & {$[0,1,2,3]+[4,5,6]$} \\
\hline 10 & 5 & 4 & {$[0,1,2,3]+[4,5,6]+[7,8,9]$} \\
\hline 11 & 5 & 4 & {$[0,1,2,3]+[4,5,7,8]+[6,9,10]$} \\
\hline 14 & 5 & 4 & {$[0,1,2,3]+[4,5,6,7]+[8,9,10]+[11,12,13]$} \\
\hline 15 & 5 & 4 & {$[0,1,2,3]+[4,5,7,9]+[6,8,10,11]+[12,13,14]$} \\
\hline 16 & 5 & 4 & {$[0,1,2,3]+[4,5,6]+[7,8,9]+[10,11,12]+[13,14,15]$} \\
\hline 17 & 5 & 4 & {$[0,1,2,3]+[4,5,6,7]+[8,10,9]+[11,12,13]+[14,15,16]$} \\
\hline 18 & 5 & 4 & {$[0,1,2,3]+[4,5,6,8]+[7,9,10,11]+[12,13,14]+[16,15,17]$} \\
\hline 19 & 5 & 4 & {$[0,1,2,3]+[4,5,6,7]+[8,9,11,12]+[10,13,14,15]+[16,17,18]$} \\
\hline 20 & 5 & 4 & {$[0,1,2,3]+[4,5,6,7]+[8,9,10]+[11,12,13]+[14,15,16]+[17,18,19]$} \\
\hline 21 & 5 & 4 & {$[0,1,2,3]+[4,6,5,7]+[8,9,10,13]+[11,12,14]+[15,16,17]+[18,19,20]$} \\
\hline 22 & 5 & 4 & {$[0,1,2,3]+[6,5,7,8]+[9,10,11,13]+[12,14,15,4]+[16,17,18]+[19,20,21]$} \\
\hline
\end{tabular}




\section{Biostatistics and Biometrics Open Access Journal}

\begin{tabular}{|c|c|c|c|}
\hline 23 & 5 & 4 & {$[0,1,2,3]+[6,5,7,8]+[9,10,11,13]+[12,14,15,16]+[4,17,18,19]+[20,21,22]$} \\
\hline 24 & 5 & 4 & {$[0,1,2,3]+[6,5,7,8]+[9,10,11,12]+[13,14,15]+[4,16,17]+[18,19,20]+[21,22,23]$} \\
\hline 25 & 5 & 4 & {$[0,1,2,3]+[6,5,7,8]+[9,10,11,12]+[13,14,15,4]+[16,17,18]+[19,20,21]+[22,23,24]$} \\
\hline$v$ & $p_{1}$ & $p_{2}$ & Sets of Shifts \\
\hline 26 & 5 & 4 & {$[0,1,2,3]+[6,5,7,9]+[8,10,11,12]+[13,14,15,16]+[17,18,19,4]+[20,21,22]+[23,24,25]$} \\
\hline 27 & 5 & 4 & {$[0,1,2,3]+[6,5,7,8]+[9,10,11,12]+[13,15,14,16]+[17,18,20,19]+[21,23,22,4]+[24,25,26]$} \\
\hline 28 & 5 & 4 & {$[0,1,2,3]+[6,5,7,8]+[9,10,11,12]+[13,14,15,16]+[17,18,20]+[4,19,21]+[22,23,24]+[25,26,27]$} \\
\hline 29 & 5 & 4 & {$[0,1,2,3]+[6,5,7,8]+[9,10,11,12]+[13,14,17,15]+[16,18,20,19]+[4,21,22]+[23,24,25]+[26,27,28]$} \\
\hline 30 & 5 & 4 & {$[0,1,2,3]+[6,5,7,8]+[9,10,12,11]+[13,14,17,15]+[16,18,20,19]+[21,22,23,4]+[25,24,26]+[27,28,29]$} \\
\hline 31 & 5 & 4 & {$[0,1,2,3]+[6,5,7,8]+[9,10,11,12]+[14,13,17,15]+[16,18,20]+[4,19,21]+[22,23,24]+[25,26,27]+[28,29,30]$} \\
\hline 32 & 5 & 4 & {$[0,1,2,3]+[6,5,7,8]+[9,10,11,12]+[15,14,17,13]+[16,18,20,19]+[21,22,23]+[4,24,25]+[26,27,28]+[29,30,31]$} \\
\hline 33 & 5 & 4 & {$[0,1,2,3]+[6,5,7,8]+[11,9,10,12]+[13,14,17,15]+[16,18,20,19]+[21,22,24,4]+[23,25,26]+[27,28,29]+[30,31,32]$} \\
\hline 34 & 5 & 4 & {$[0,1,2,3]+[6,5,7,8]+[9,11,10,12]+[13,14,17,15]+[16,20,18,19]+[21,22,23,24]+[25,26,27,4]+[28,29,30]+[31,32,33]$} \\
\hline 35 & 5 & 4 & {$[0,1,2,3]+[6,5,7,8]+[9,11,10,12]+[13,14,17,15]+[16,18,20,19]+[21,22,23,24]+[25,26,27,28]+[29,30,31,32]+[4,33,34]$} \\
\hline 36 & 5 & 4 & {$[0,1,2,3]+[6,5,7,8]+[9,11,10,12]+[13,14,17,15]+[16,18,20,19]+[21,22,23,24]+[25,26,27]+[4,28,29]+[30,31,32]+[33,34,35]$} \\
\hline 37 & 5 & 4 & {$[0,1,2,3]+[6,5,7,8]+[9,11,10,12]+[13,14,17,15]+[16,18,20,19]+[21,22,23,24]+[25,26,27,4]+[28,29,30]+[31,32,33]+[34,35,36]$} \\
\hline 38 & 5 & 4 & {$[0,1,2,3]+[6,5,7,8]+[9,11,10,12]+[13,14,17,15]+[16,18,19,20]+[21,22,23,24]+[25,26,27,28]+[29,30,31,4]+[32,33,34]+[35,36,37]$} \\
\hline 39 & 5 & 4 & $\begin{array}{l}{[0,1,2,3]+[6,5,7,8]+[9,11,10,12]+[13,14,17,15]+[16,19,18,20]+[21,22,23,24]+[25,26,28,27]+[29,30,31,32]+[4,33,34,35]+[36,37,3} \\
8]\end{array}$ \\
\hline 40 & 5 & 4 & $\begin{array}{l}{[0,1,2,3]+[6,5,7,8]+[9,11,10,12]+[13,14,17,15]+[16,19,18,20]+[21,22,23,24]+[25,26,27,28]+[29,30,31]+[4,32,33]+[34,35,36]+[3} \\
7,38,39]\end{array}$ \\
\hline 41 & 5 & 4 & $\begin{array}{l}{[0,1,2,3]+[6,5,7,8]+[9,11,10,12]+[13,14,17,15]+[16,19,18,20]+[21,22,23,24]+[25,26,27,28]+[4,29,30,31]+[32,33,34]+[35,36,37]} \\
+[38,39,40]\end{array}$ \\
\hline 42 & 5 & 4 & $\begin{array}{l}{[0,1,2,3]+[6,5,7,8]+[9,11,10,13]+[12,14,17,15]+[16,19,18,20]+[21,22,23,24]+[25,26,27,28]+[29,30,31,32]+[4,33,34,35]+[36,37,3} \\
8]+[39,40,41]\end{array}$ \\
\hline 43 & 5 & 4 & $\begin{array}{l}{[0,1,2,3]+[6,5,7,8]+[9,11,10,12]+[13,14,17,15]+[16,19,18,20]+[21,22,23,24]+[25,26,27,28]+[29,30,31,32]+[33,34,35,36]+[4,37,3} \\
8,39]+[40,41,42]\end{array}$ \\
\hline 44 & 5 & 4 & $\begin{array}{l}{[0,1,2,3]+[6,5,7,8]+[9,11,10,12]+[13,14,15,16]+[17,19,18,20]+[21,22,23,24]+[25,26,27,28]+[29,30,31,32]+[33,34,35]+[4,36,37]} \\
+[38,39,40]+[41,42,43]\end{array}$ \\
\hline 45 & 5 & 4 & $\begin{array}{l}{[0,1,2,3]+[6,5,7,8]+[9,11,10,12]+[13,14,15,17]+[16,19,18,20]+[21,22,23,25]+[24,26,27,28]+[29,30,32,31]+[33,34,35,4]+[36,37,} \\
38]+[39,40,41]+[42,43,44]\end{array}$ \\
\hline 46 & 5 & 4 & $\begin{array}{l}{[0,1,2,3]+[6,5,7,8]+[9,11,10,12]+[13,14,15,16]+[17,19,18,20]+[21,22,23,25]+[24,26,27,28]+[29,30,32,31]+[33,34,35,37]+[4,36,3} \\
8,39]+[40,41,42]+[43,44,45]\end{array}$ \\
\hline 47 & 5 & 4 & $\begin{array}{l}{[0,1,2,3]+[6,5,7,8]+[9,11,10,12]+[13,14,15,16]+[17,19,18,20]+[21,22,23,25]+[24,26,27,28]+[29,30,32,31]+[33,34,35,36]+[37,38,} \\
39,40]+[4,41,42,43]+[44,45,46]\end{array}$ \\
\hline$v$ & $p_{1}$ & $p_{2}$ & Sets of Shifts \\
\hline 48 & 5 & 4 & $\begin{array}{l}{[0,1,2,3]+[6,5,7,8]+[9,11,10,12]+[13,14,15,16]+[17,19,18,20]+[21,22,23,25]+[24,26,27,28]+[29,30,32,31]+[33,34,35,36]+[37,38,} \\
39]+[4,40,41]+[42,43,44]+[45,46,47]\end{array}$ \\
\hline 49 & 5 & 4 & $\begin{array}{l}{[0,1,2,3]+[6,5,7,8]+[9,11,10,12]+[13,14,15,16]+[17,19,18,20]+[21,22,23,25]+[24,26,27,28]+[29,30,32,31]+[33,34,35,36]+[37,38,} \\
39,40]+[4,41,42]+[43,44,45]+[46,47,48]\end{array}$ \\
\hline 50 & 5 & 4 & $\begin{array}{l}{[0,1,2,3]+[6,5,7,8]+[9,11,10,12]+[13,14,15,16]+[17,19,18,20]+[21,22,23,25]+[24,26,27,28]+[29,30,32,31]+[33,34,35,36]+[37,38,} \\
39,40]+[41,42,43,4]+[44,45,46]+[47,48,49]\end{array}$ \\
\hline
\end{tabular}

\section{References}

1. Iqbal I, Tahir MH (2009) Circular strongly balanced repeated measurements designs. Communications in Statistics-Theory and Methods 38(20): 3686-3696.

2. Williams ER (1949) Experimental designs balanced for the estimation of residual effects of treatments. Australian Journal of Science Research A2: $149-168$

3. Williams EJ (1950) Experimental designs balanced for pairs of residual effects. Australian Journal of Science Research 3(3): 351-363.
4. Magda CG (1980) Circular balanced repeated measurements designs Communications in Statistics-Theory and Methods 9(18): 1901-1918.

5. Roy BK (1988) Construction of strongly balanced uniform repeated measurements designs. Journal of Statistical Planning and Inference 19(3): 341-348.

6. Jones B, Kenward MG (1989) Design and Analysis of Cross Over Trials. London: Chapman and Hall.

7. Afsarinejed K (1990) Repeated measurements designs-a review. Communication in Statistics- Theory and Methods 19(11): 3985-4028. 
8. Cheng CS, Wu CF (1980) Balanced repeated measurements designs. Ann Statist 8(6): 1272-1283.

9. Iqbal I, Jones B (1994) Efficient repeated measurements designs with equal and unequal period sizes. Journal of statistical planning and inference 42(1): 79-88.

10. Sharma VK, Jaggi S, Varghese C (2003) Minimal balanced repeated measurements designs. Journal of Applied Statistics 30(8): 867-872.
11. Iqbal I, Tahir MH, Ghazali SA (2010) Circular first-and second-order balanced repeated measurements designs. Communications in Statistics-Theory and Methods 39(2): 228-240.

12. Hedayat AS, Yang M (2003) Universal optimality of balanced uniform crossover designs. Ann Statist 31(3): 978-983.

\section{Your next submission with Juniper Publishers will reach you the below assets}

- Quality Editorial service

- Swift Peer Review

- Reprints availability

- E-prints Service

- Manuscript Podcast for convenient understanding

- Global attainment for your research

- Manuscript accessibility in different formats

( Pdf, E-pub, Full Text, Audio)

- Unceasing customer service

Track the below URL for one-step submission https://juniperpublishers.com/online-submission.php 